\title{
Tracheal Obstruction, CTCAE
}

National Cancer Institute

\section{Source}

National Cancer Institute. Tracheal Obstruction, CT CAE. NCI Thesaurus. Code C143882.

A disorder characterized by blockage of the lumen of the trachea. 